\title{
Visualising social computing output: Mapping student blogs and tweets
}

\author{
David Cameron
}

University of Newcastle, Australia

david.cameron@newcastle.edu.au

\section{Amalie Finlayson}

Charles Sturt University, Australia

afinlayson@csu.edu.au

\section{Rebecca Wotzko}

Charles Sturt University, Australia rwotzko@csu.edu.au

\section{Introduction}

This chapter provides a case study in the development of a data mining approach to assess blogging and micro-blogging (or 'tweets') in a higher education setting. Data mining is the use of computational algorithms to analyse large datasets, and this chapter describes the use of the Leximancer software tool to perform a conceptual analysis of the blogs and tweets published by students in an undergraduate course about social media. A Leximancer analysis is represented visually as a 'concept map' showing the relationships between the concepts and ideas drawn out of the data automatically, rather than using pre-defined terms and keywords. In this chapter, Leximancer is used to produce a concept map of the student blogs and tweets to enhance evaluation of the students' conceptual understanding of the syllabus, as well as more general observations about the use of these social media tools in higher education. This suggests a possible approach to analysing the potentially large volume of text-based information that can be produced by students in these social computing settings. 


\section{Teaching Social Media with social media}

Social computing technology is now constantly evolving in a process described as 'perpetual beta', in which it is "developed in the open, with new features slipstreamed in on a monthly, weekly, or even daily basis" (O'Reilly 2005). Teachers working with these tools can find themselves also constantly evaluating, maintaining and updating subject matter in a similar perpetual beta model (Finlayson et al. 2009). In addition, the assumption that so-called 'digital native' learners would have a natural and homogeneous affinity for using these technologies in their education has increasingly been questioned (Kennedy et al. 2008). For example, the authors have noted significant variations in skills and comfort levels with different applications, and a tendency for students to equate their frequent but often unsophisticated use of some sites, such as Facebook, with mastery of social media generally. This self-assessment can lead to attitudes of resistance from some learners when they are asked to apply these tools in educational settings. A third factor for consideration is that the ease with which content can be produced with these tools can potentially leave educators and researchers with a large volume of student output to review or assess. Increasingly sophisticated data mining and visualisation tools and techniques are emerging to explore these data sets (for example Java et al. 2007).

At Charles Sturt University the course titled COM340 Social Media is delivered to undergraduate Communication and Media students, with the aim of engaging them with the potential professional applications of social computing tools such as social networks (e.g. Facebook), blogs, wikis, micro-blogs (e.g. Twitter), and mobile media. The 2010 cohort of students in COM340 Social Media was introduced to concepts and practice around social media, with an emphasis on the journalism and public relations industries. The students were required to use blogging and micro-blogging tools to report on their own use of and attitudes towards Web 2.0 and social media applications, and to reflect on the potential impact of these technologies on their future careers in the media and communication industries. Many also chose to comment on the integration of social media into the course's delivery, providing a critique of the syllabus and the teaching and learning strategies.

\section{The CSU Interact Collaboration and Learning Environment}

The COM340 Social Media course is delivered simultaneously to both on-campus (face-to-face) and distance education (online) cohorts. Charles Sturt University's teaching and learning strategies are focused on a collaborative learning environment (CLE), called CSU Interact, which is based on the open source Sakai project 
(http://sakaiproject.org/portal). CSU Interact is a mandatory teaching resource for all courses taught at Charles Sturt, including those still considered to be delivered primarily in a face-to-face mode. The CLE provides a default set of online tools such a discussion forum, course outline, announcements, a course evaluation survey and electronic assignment submission. Lecturing staff may then select additional online teaching and learning tools from a suite of options including a weblog, a wiki, lesson modules, RSS feeds, links to external websites, chat, eportfolios and file-sharing. The tool used for COM340 Social Media in 2010 and discussed in this chapter is the blog publication tool integrated into the Sakai CLE. In addition, the course made use of the external micro-blogging application Twitter (www.twitter.com).

\section{The Blogging tool and task}

The CSU Interact online environment contains a blog tool that students use as a reflective learning journal. In COM340 Social Media, students used this tool for an assessment task requiring them to:

- post regular entries to their own personal weblog, hosted within the CSU Interact site for COM340, focusing on content related to social media;

- focus on producing quality, compelling and rich web content as well as a regular quantity of content;

- utilise the blog as a space to engage with weekly reflection on readings and the course content; and

- provide critical feedback and comment on other students' blog posts.

Students used the CSU Interact blog tool in class to document the completion of tutorial activities, and outside of class to reflect on the course content. It was hoped that the simplicity of the blog tool would make it easy for students to create posts, upload their own media, and link to external media content.

\section{Micro-blogging task via Twitter}

In addition to the blog assessment task, the instructors decided to explore the emerging principles and practice of micro-blogging as they related to the journalism and public relations industries. Twitter was chosen due to its widespread uptake and use in the communication industry.

The introduction of Twitter to the course was initially met with resistance from the 2010 cohort of students. A survey of their social media use at the beginning of the 
course indicated that almost $70 \%$ of students had never used Twitter, with many respondents also indicating that they were not planning to ever use it. However, students were required to sign up for an account to participate in a collaborative lecture focused on the topic of blogging and micro-blogging. This environment allowed students to experiment with Twitter communication conventions, and to contribute to an ongoing conversation about the Social Media course by using the 'hashtag' (a keyword that categorises tweets) '\#com340'.

The Twitter tasks were not set as summative assessment tasks, in part due to privacy, intellectual property and academic governance issues that might be raised by forcing students to make use of an externally hosted online publication medium. While we acknowledge that there are advantages to be gained by engaging students with social computing applications 'in the wild', it was not considered crucial for the learning objectives of this course. For similar reasons, it was decided to make use of the internally hosted blog tool within the CSU Interact CLE, although throughout the course students were exposed to externally hosted blogs as examples of current practice.

\section{Methodology}

Our objective in this small study was to explore the application of a data mining approach to evaluating student engagement with the syllabus, as reflected in their course-related blog and micro-blogging output.

The methodology for this chapter falls into the qualitative paradigm and utilises a 'reflective practitioner' or autoethnographic perspective, on the basis that autoethnographies are recognised as providing a solid footing for qualitative research in the social sciences (Hustler 2005).

Further, in this study, the use of a grounded theory approach is used to enable a theoretical stance to be developed from the data (Strauss and Corbin 1990). Given that theory-generation is the principle aim (Corbin 2005), the research in this study was not conducted with a predefined theory in mind, as the rapid change and ongoing developments in the area of social media education mean that there is as yet little theoretical or conceptual apparatus to draw on when conducting studies of this kind. Importantly, therefore, careful delimitation was also a part of the methodological process. The data in this instance is limited, as a result, to that generated within a specific context: student blogging and micro-blogging output for the COM340 course in 2010. The sample was further refined to include only those students studying in the on-campus mode, as they were engaged more directly with the blogging and micro-blogging exercises through lecture and tutorial content. 
Multiple forms of data in the form of raw text from both student blogs and student tweets were collected and analysed for this case study, primarily using the opencoding software analysis tool known as Leximancer. Visual representations of this data, or 'themic maps', along with lists of primary themes relating to this data, were then created.

\section{Leximancer}

Leximancer is a software tool used to find meaning in text-based documents. It automatically identifies key themes and ideas by data-mining texts, and visually representing information in 'themic maps', showing the relationships between these themes and ideas. In these maps, the thematic groups are surrounded by colourcoded circles, where the size of the circle indicates the frequency of the occurrence of the theme throughout the data, and the position of the circle indicates the relationship of that theme to the other themes.

This form of textual analysis was identified as suitable for this study as it adds a quantitative, scientific analysis component to the method. The benefits of using the Leximancer tool are evident: as a form of analysis it is highly inclusive as all articles within the text are treated as important. Every article contributes to the analysis and to overall understanding. The picture that emerges from this analysis is, in essence, a user-driven representation of meaning.

Leximancer is a commercial product, though a number of pricing options are provided for academic use. The authors are not aware of any open source alternatives that provide the same functionality.

\section{Concept mapping}

The concepts that Leximancer produces in the primary themic maps are generated through thousands of iterations, as the software observes clusters of words that move with key terms in the data. As Leximancer identifies some words appearing frequently with certain terms and less frequently with others, the software 'learns' that the words that travel together with a term, frames the profile of that term and defines that term to be a key theme in the data.

Another feature of Leximancer is that "it also allows the user to request overviews of text sources and key segments of texts in relation to specific concepts and relationships between concepts" (Fisk et al.). This feature was seen as a highly useful 
facet of the Leximancer data mining approach, as it helped single out text sources and key segments of texts in relation to specific concepts under discussion without needing to search through large samples of student blog and micro-blog text.

The authors therefore worked with the highly inclusive analysis tool Leximancer, coupled with their own conceptual sensitivity and practical experiential knowledge, to identify themes and formulate valid and representative themic maps based on the blogging and micro-blogging output of students enrolled in the COM340 course for 2010 .

\section{Collecting and processing the data}

Two data sets were collected for the purposes of this study: the blog content produced by students as part of their work in the COM340 session for 2010, and the micro-blog or 'tweet' content produced by students on the Twitter micro-blogging application for the same COM340 session. For this study the authors collected both sets of data manually by copying and pasting the content into text files, in preparation for analysis using the Leximancer tool.

In order to comply with ethical considerations, no individual names or aliases were included in the analytical output of the study, so as to guarantee students' anonymity. The omission of names in this way was a deliberate act, in recognition of the tension that currently exists in new forms of digital publishing (such as blogs and micro-blogs) around the public/private nature of information made available by individuals in these mediums (Viégas 2005). Indeed, one of the key learning objectives of COM340 is to raise students' awareness of the personal risks of publishing in these "networked publics" (Boyd 2007).

\section{Results}

All of the blog text was grouped into one data set, and all of the micro-blog or tweet text into another, so as to create two separate data sets for analysis by Leximancer. These were then run through the analysis process to create a separate concept map for each. 


\section{Concept map of student blogs}

The analysis of the student blogs was initially conducted by bundling all of the student blog content together. For the purposes of the study, the sample was limited to the 60 students who completed the course in the on-campus mode. The content of all 60 blogs was treated as one set of data, and running all the text from these created an initial exploratory map.

Few of the pre-processing options allowed by Leximancer were adjusted in the initial phase of analysis. The prose test threshold, which usually defaults to a value of one to allow the software to skip parts of the text that it sees as redundant or extraneous, was reduced to zero to ensure that Leximancer checked every single part of the blog texts under analysis. The default setting suits analysis of texts that are comprised of full sentences in a prose format, as it would be advisable to ignore any sentences that are not full prose sentences. However, as this particular set of text data contained student blog entries of more casual or informal language, the prose test threshold was removed.

In the Automatic Concept Identification options, it was decided to allow Leximancer to automatically identify the concepts existing within the data set of the text of the 60 student blogs. All other settings were also left on the default setting at this point.

An initial themic map was then generated, with a view to having something to refer to and alter so as to produce a final, relevant map or data visualisation. After consulting this initial resulting map, and the list of themes that the software generated automatically from the blog text data, some unnecessary and redundant concepts were removed. It was necessary to delete some concepts and merge others in the primary themic maps as the data under analysis was, as described above, produced by analysis of the text of a collection of student blog entries. As such, the data contained several informal elements: words that were adjectival, for example, or that were used as part of the casual vernacular of students, or which were simply irrelevant. These words included 'able', 'access', 'Bathurst', 'become', 'best', 'check', 'day', 'example', 'fact', 'group', 'interesting', 'look', 'name', 'post', 'probably', 'someone', 'sure', 'take', 'things', 'thought', 'use', 'used', 'users', 'using', 'via' and 'year'.

Further review revealed that some other Leximancer-identified words or concepts belonged together or were very similar to each other, and could be merged so as to create a simpler and clearer themic map. It was necessary to manually merge these concepts within the data - these included 'social' and 'media', 'friend' and 'friends', 'people' and 'person' and 'site', 'sites' and 'websites'. 
After these changes had been made in terms of the instructions for how Leximancer was to analyse the data set, the second and final map was more stable. These general maps and broad analysis techniques represented the initial stage of analysis for this part of the student blog data set.

From this point, it was possible to generate a useful and viable map to help to identify the major themes within the data, as part of the grounded theory methodology identified earlier in this section. This is shown as Figure 1.

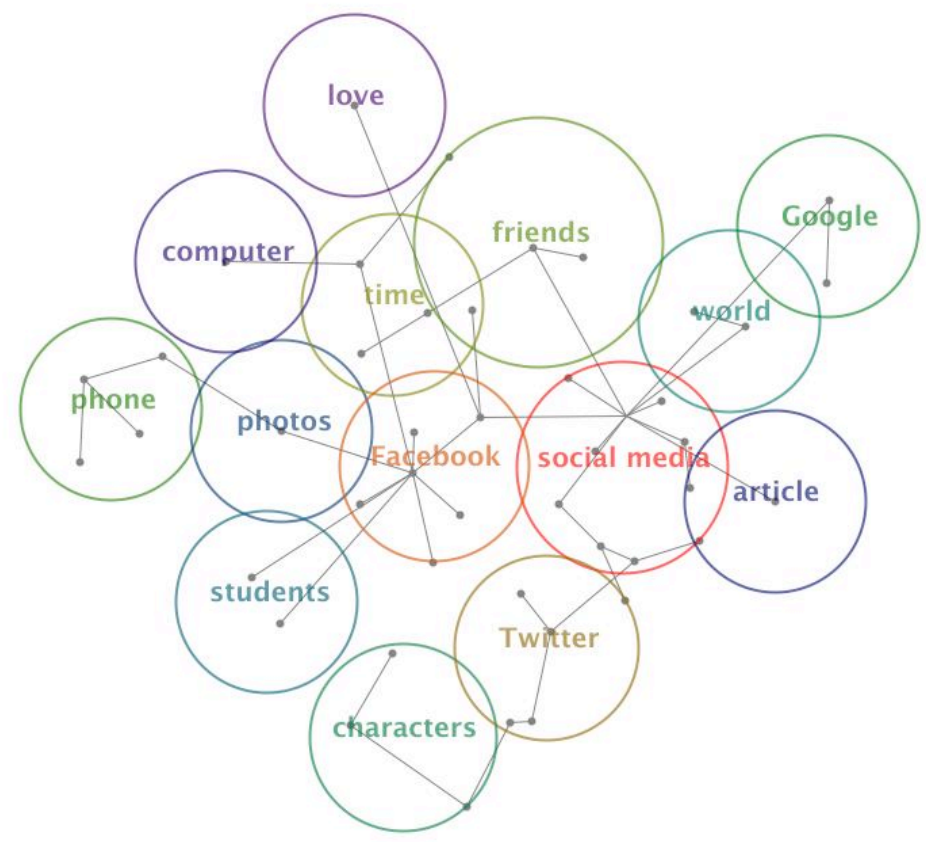

Figure 1: Leximancer map - primary themes in student blogs $(n=60)$

The concept map provides a starting point for the researcher to begin generating theories from the data. In Figure 1 it can be seen that 'social media' is a major theme, due its relative size. However, in a colour map it is presented as red, which indicates that it is possibly less significant to the researcher than, for example, the smaller themes of Facebook and Twitter which are in hues more towards the blue end of the spectrum. This is actually quite intuitive, as the theme of social media could be expected to be common across the data set due to the name and content of the course, and the frequency therefore that these terms are used in the student blogs. At an initial glance, the theme of 'friends' could be considered more worthy 
of further investigation, as it is indicated as significant in the data set through both size and colour coding (it is presented as a green/blue hue).

For the researcher, the concept map can therefore provide the launching point for other tools within Leximancer. The next step in this study was to consider the concept map in parallel with Leximancer's list of the main themes identified as applicable to this unique student blog data set. These themes were organised in order of connectivity, or relevance, and the percentage next to them indicates the level of connectivity or relevance. That is, the top theme, social media, was chosen as the most relevant theme of all the available themes from the data, and was scaled as being $100 \%$ connected. Leximancer scaled all the other themes accordingly.

Table 1: Themes and connectivity for student blogs $(n=60)$

\begin{tabular}{|l|l|}
\hline Theme & Connectivity \\
\hline Social media & $100 \%$ \\
\hline Facebook & $69 \%$ \\
\hline Twitter & $23 \%$ \\
\hline Time & $19 \%$ \\
\hline Friends & $19 \%$ \\
\hline Phone & $14 \%$ \\
\hline Google & $14 \%$ \\
\hline Characters & $10 \%$ \\
\hline World & $9 \%$ \\
\hline Students & $5 \%$ \\
\hline Photo & $4 \%$ \\
\hline Article & $2 \%$ \\
\hline Computer & $2 \%$ \\
\hline Love & $2 \%$ \\
\hline
\end{tabular}

As can be seen in Table 1, 'social media' was identified as by far the most relevant theme for this student blog data set with $100 \%$ relevance or connectivity, followed by 'Facebook' at $69 \%$, 'Twitter' at $23 \%$, 'time' at $19 \%$, 'friends' at $19 \%$, 'phone' at $14 \%$, 'Google' at $14 \%$, 'characters' at $10 \%$, 'world' at $9 \%$, 'students' at $5 \%$, 'photos' at $4 \%$, 'article' at $2 \%$, 'computer' at $2 \%$ and 'love' at $2 \%$.

The top three themes were not surprising to the authors given their own knowledge of the topics and the subject matter of the course, and this provides a simple check of the validity of the Leximancer output. However, as noted earlier in the discussion on colour coding in the concept map, these ranking alone do not necessarily offer a guide as to the nature of these themes. The third step in this Leximancer analysis of student blogs was to use Leximancer's linking feature that 
enables the researcher to click into examples of the themes and concepts from the data set, to investigate at a highly granular level the contexts in which the terms are used. By moving between concept map, theme rankings and examples of use in this way, a clearer picture of the emerging themes can be quickly developed from a large data set.

\section{Concept map of student tweets}

The analysis process for the student micro-blogs or tweets was initially conducted within Leximancer by once again bundling all of the student micro-blog blog content together. For the purposes of the study, the number of students who engaged with the Twitter application numbered much lower at 35 students, presumably because this was not an assessable part of student output for the COM340 course in 2010. Therefore, for this part of the analysis, the quantity of student blogs included was $n=35$. The manually copied content of all 35 Twitter streams was treated as one set of data, and running all the text from these 35 Twitter streams created an initial exploratory map.

Once again, only the minimum Leximancer settings were adjusted in the initial phase of analysis. The prose test threshold was again reduced to zero to ensure that Leximancer checked every single part of the micro-blog text under analysis for this part of the study. Leximancer was again allowed to automatically identify the concepts existing within the data set, and all other settings were also left at the default level.

A working themic map was then generated, and then some unnecessary and redundant words or concepts were removed. These words included 'amazing', 'best', 'bring', 'coming', 'feel', 'makes', 'RT', 'use', 'used', 'via', 'wait', 'watching' and 'year'. For this data set, just two words or concepts were merged: 'social' and 'media'.

A more relevant map was then generated for use by the authors to help identify the major themes and the relationship of these themes, and can be seen below in Figure 2. 


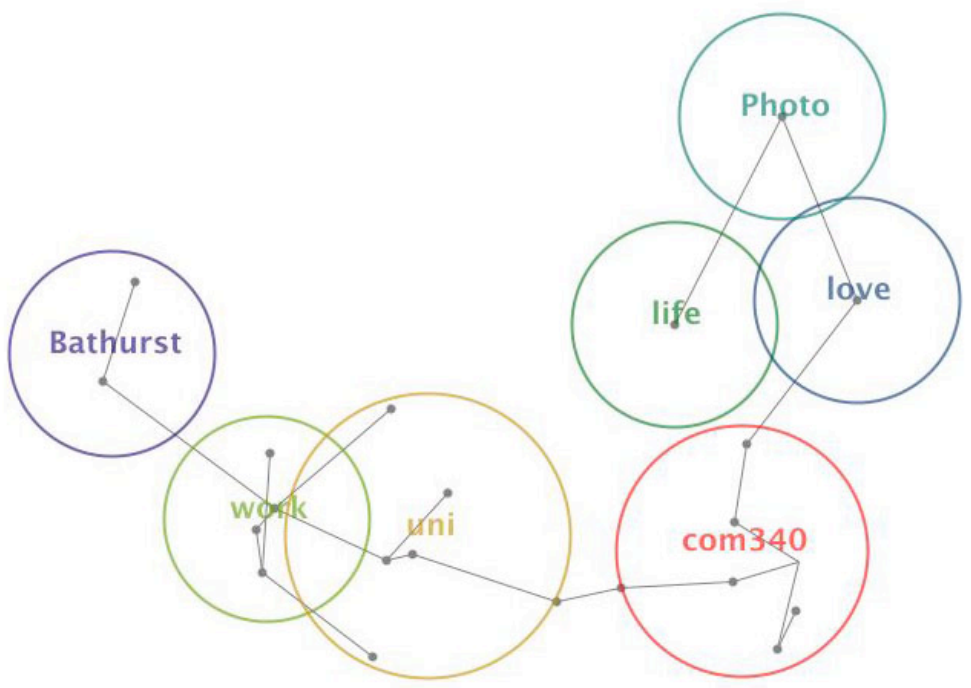

Figure 2: Leximancer concept map - primary themes identified in the student micro-blogs $(n=35)$

Leximancer also produced a list of the major themes that were applicable to this unique student micro-blog or tweet data set. The top theme, social media, was chosen as the most relevant theme of all the available themes from the data, and was scaled as being $100 \%$ connected. Leximancer scaled all the other themes accordingly.

Table 2: Leximancer themes and connectivity for student micro-blogs $(n=35)$

\begin{tabular}{|l|l|}
\hline Theme & Connectivity \\
\hline COM340 & $100 \%$ \\
\hline Uni & $60 \%$ \\
\hline Work & $35 \%$ \\
\hline Life & $7 \%$ \\
\hline Photo & $7 \%$ \\
\hline Love & $6 \%$ \\
\hline Bathurst & $6 \%$ \\
\hline
\end{tabular}


In this data set, 'COM340' was the most relevant theme with $100 \%$ relevance or connectivity, followed by 'uni' at $60 \%$, 'work' at 35\%, 'life' at 7\%, 'photo' at 7\%, 'love' at $6 \%$ and 'Bathurst' at $6 \%$.

The COM340 theme was not unexpected by the authors, again due to the likely subject matter of the tweets. However, the other top themes of 'uni' and 'work' had not been anticipated, which illustrates how Leximancer can point investigators to unexpected themes emerging from a data set. At this stage, having generated a broad themic map and list and a more specific concept list for both the blog and micro-blog data sets, the conceptual data was further interrogated.

\section{Discussion}

As discussed earlier, the Leximancer concept map provides a visualisation of the relevance and connectivity of themes and concepts emerging from the text data, however more detailed investigation is then required to explore the context in which these themes appear within the data set. Leximancer does not provide 'answers', but instead provides a set of tools to help the researcher traverse the data set in search of meaning. An initial observation of the concept maps and theme rankings produced in this study shows that the student reflections centred on 'social media', 'Facebook', 'Twitter' and 'COM340'. At a casual level these would not be unexpected given the subject matter, but drilling down to some sample examples of usage draws the researcher to arrange of interesting observations.

Conversely, deeper examination of less obvious themes such as 'friends', 'article', 'characters' and 'love' revealed that they often referred to the outputs of one-off class exercises and were therefore less relevant to the investigation. For example, in a tutorial the students were asked to devise a proposal for a transmedia drama that used social media to tell a dramatic narrative. Their subsequent blog reflections therefore made frequent mention to the terms 'characters' and 'friends' in describing their plot synopses. Another tutorial prompted students to reflect on a reading, which boosted the perceived significance of the concept of 'article'. The theme of 'love' emerged from the students' common use of the term in a verbal form to indicate a liking or preference for something, as in these examples:

\footnotetext{
"I'm pretty sure that after a big night people love going online and checking out what happened the night before" (Student 1, blog post).

"I'd love to hear from all my fellow COM340ers and your experiences with twitter" (Student 2, blog post).

"Would love to have a shower with hot water for once and some heating. Damn \#Uni!" (Student 3, tweet).
} 


\section{Students' conceptual understanding of the syllabus}

From the start of the course it was clear that many students perceived COM340 Social Media as 'the Facebook course'. This was partly reinforced by the timetabling of a two-hour tutorial each week in a computer laboratory, which placed social media tools at the students' fingertips and proved both a convenience and distraction for teaching. Also apparent was the students' general resistance to Twitter, which was revealed in a survey of their social media habits undertaken at the start of the course and then reflected in their class discussions, blog posts and tweets. A typical comment was:

"If everybody is connected to facebook do I really need to get my head around twitter? Apparently so, if only for the lecture for this subject in two weeks" (Student 4, blog post).

However, the ability of Leximancer to link themes with contextual examples in the data set allowed the authors to identify moments of direct engagement with the syllabus. Student blogs would reflect directly on issues raised in the weekly lectures and tutorial sessions, such as the use of Twitter by journalists trying to track a breaking news story:

"I was at work in a radio newsroom when Michael Jackson died and found Twitter especially useful during this busy morning ... I used a variety of tweets to find credible sources" (Student 5, blog post).

Other examples of direct reflection on the syllabus included students' posts on privacy issues in social networking sites, and the topic of online memorials:

\footnotetext{
"Although education is important I think the creators of sites like Facebook need to take greater responsibility" (Student 6 , blog post).

"I think that i would definitely prefer it if my social media footprint was deleted. While my social media sites are fairly secure and don't allow strangers to see my information, there are still things hidden away on my Facebook page that i don't think i would like other people to see, or have access to" (student 7, blog post).
}

Another observation that can be made from comparing the two data sets is that the bulk of tweets from the COM340 students could be categorised as personal rather than course-focused. This can in part be explained by the fact that the tweeting exercise was not assessable, and that the students' had been quite clearly told to use the blog as the main platform for their reflections. For the majority of students, as noted in a survey conducted at the start of the course, this was also the first time they had used Twitter and so many of their tweets had an air of being guarded, or superficially exploring how the application works. This was interesting to the authors, given that the student resistance to Twitter was often articulated in terms of 
it being a channel for irrelevant and banal personal observations. Ironically they failed to perceive the same elements in their own tweets, such as:

\footnotetext{
"So... I went out last night, and got home@ @11am today - Instead of doing work" (Student 8, tweet).

"Check out the fog that has swept over Bathurst this morning... Winter is on its way!" (Student 9, tweet).
}

\section{Students' reflections on their own social media use}

A key teaching strategy used in COM340 Social Media was to encourage students to evaluate and reflect on their own use of social media tools, and to compare that with the defined demographics of some of the more popular applications. The course also focused on the use of social media tools by media and communication industries. The aim of this strategy was to help students acknowledge their own often heavily biased - perceptions of social media as consumers, and engage with broader concepts as producers and content creators. From the Leximancer examination of the blog and tweet data sets, and triangulated with other sources such as tutorial discussions and other tasks in the course, it was clear that this strategy had mixed results with this cohort. Some students clearly had difficulty overcoming their personal preferences for certain applications, as evidenced in the superficial 'Facebook versus Twitter' debates that dominated their blog posts. Their reflections were also heavily based on their own current usage at a consumer level, rather than their application or potential usage by media or communication content producers: Typical of this introspective commentary are these reflections:

\footnotetext{
"There are certain aspects of Facebook that I am simply not interested in using, for example facebookchat, and 'become a fan of'. I am also quite hesitant to join certain groups, for example Political or Religious advocacy groups" (Student 10, blog post).

"I use Twitter to tweet randomly when I'm in a class with a computer or if I'm stuck late at the uni and bored. I'm only following 2 other people and they tweet rarely as well" (Student 11, blog post).
}

\section{Students' observations on the use of social media tools in higher education}

The students' reflections on the subject content of COM340 inevitably included commentary on the delivery of the course itself. These did include some positive observations, particularly from those students who made a connection between social media and their career aspirations in the media and communication industries. As noted above there was at times bitter resistance to the idea that the course would require students to examine and use micro-blogging, or more particularly, 
Twitter. Educators need to be wary of assuming that all social media tools are equally popular, or that students will automatically be willing experimenters with online tools. These students clearly had difficulty detaching themselves from their personal social media habits. While a handful of students were already prolific Twitter users, the majority indicated little or no experience, and some were opposed to trying it at all:

\section{"YOU WANT ME TO JOIN TWITTER!? THE SAME TWITTER THAT I'VE BEEN MOCKING AND FIGHTING AGAINST SINCE IT FIRST STARTED?” (Student $\mathrm{x}$, tweet).}

Along similar lines, the heavy use of online resources in a face-to-face setting produced some comments from students. It was clear that some felt that the requirement to attend face-to-face classes that demonstrated the affordances of social media tools did not make sense, as with this tweeted note:

\footnotetext{
"I'm at uni, in the maclabs, for a lecture being delivered by Twitter. Why couldn't I do this from bed?" (Student 12, tweet).
}

The Leximancer analysis also provided links to more general observations about the delivery of the course, for example the timetabling of classes was a regular source of comments:

“mmmm 8am lecture for @com340. Loving life” (Student 13, tweet).

\section{Conclusion}

The sample size used for this study is small, and we make no generalised claims about the specific experiences of these students or their engagement with the subject matter. Rather, we argue that the adoption of a data mining approach may provide educators with a set of tools and techniques to assist them when working with the large volume of output that can be generated in some social media publishing forms.

From this trial the authors found that the use of a data mining approach to student social media output provided some useful insights into student engagement with the syllabus, and the course delivery strategies. We could apply our broader knowledge of what was going on in class with the themes and concepts emerging from the data analysis, and use this to inform our reflections on the course content and delivery. Leximancer's visualisation of the themes and concepts as a graphic map was a useful starting point for our analysis of the large volume of text generated by the use of blogs and tweets in COM340 Social Media. Although we had some preconceived notions as to what the dominant themes would be in the stu- 
dent responses, the use of Leximancer to draw out meaning from the text provided a useful means by which to test our own views, and to identify unexpected themes.

The ability of Leximancer to then allow the researcher to drill down to examples of the themes and concepts in context within the data set also provided a useful means by which to explore the meaning of the themes and concepts in terms of the course as a whole. It enabled the authors to navigate through large volumes of blog and tweet text to become sensitised to the types of comments being made by the cohort about different aspects of the syllabus and the delivery of the course itself.

One area that presents itself as an area for future research is the potential use of data mining tools more directly in the assessment process. This study concentrated on a post-assessment analysis of the textual output from the students. However, the ability of software tools such as Leximancer to collate and analyse the work of individual students is worthy of further investigation. Similarly, processes for automatically collecting the output of students using blogs and micro-blogs - either hosted within a CLE or externally hosted - are also worth considering to enhance the use of data mining tools in educational settings.

\section{References}

Boyd D (2007) Why youth (heart) social network sites: The role of networked publics in teenage social life. In: Buckingham D (ed) Macarthur foundation series on digital learning: Youth, identity, and digital media volume. MIT Press, Cambridge, MA,

Corbin J (2005) Grounded theory. In: Somekh B, Lewin C (eds) Research methods in the social sciences. Sage Publications, London, Thousand Oaks, New Delhi, pp 49-55

Finlayson A, Cameron D, Hardy M Journalism education as a perpetual beta test: Notes on the design and delivery of tertiary 'social media' subjects. In: Journalism Education Association of Australia, Perth, 2009.

Fisk K, Cherney DA, Hornsey APM, Smith DA Rebuilding institutional legitimacy in post-conflict societies: An asia pacific case study - phase 1a. Air Force Office of Scientific Research (AFOSR)

Asian Office of Aerospace Research and Development (AOARD)

University of Queensland,

Hustler D (2005) Ethnography. In: Somekh B, Lewin C (eds) Research methods in the social sciences. Sage Publications, 
Java A, Song X, Finin T, Tseng B Why we twitter: Understanding microblogging usage and communities. In: Joint 9th WEBKDD and 1st SNA-KDD Workshop, 2007.

Kennedy GE, Judd TS, Churchward A, Gray K, Krause K-L (2008) First year students' experiences with technology: Are they really digital natives? Australasian Journal of Educational Technology 24 (1):108 - 122

O'Reilly T (2005) What is web 2.0? http://oreilly.com/pub/a/web2/archive/whatis-web-20.html?page $=4$. Accessed 1 August, 2010

Strauss AL, Corbin J (1990) Basics of qualitative research. Sage Publications, Newvbury Park

Viégas FB (2005) Bloggers' expectations of privacy and accountability: An initial survey. Journal of Computer-Mediated Communication 10 (3) 